\title{
De la paternité biologique à la paternité littéraire : étapes d'une argumentation chez Montaigne
}

From Biological to Literary Paternity: The Stages of an Argumentation in Montaigne

Nadine Kuperty-Tsur

\section{(2) OpenEdition}

\section{Journals}

Édition électronique

URL : http://journals.openedition.org/aad/1006

DOI : 10.4000/aad.1006

ISSN : 1565-8961

Éditeur

Université de Tel-Aviv

Référence électronique

Nadine Kuperty-Tsur, « De la paternité biologique à la paternité littéraire : étapes d'une argumentation chez Montaigne », Argumentation et Analyse du Discours [En ligne], 5 | 2010, mis en ligne le 20 octobre 2010, consulté le 10 décembre 2020. URL : http://journals.openedition.org/aad/1006 ; DOI : https:// doi.org/10.4000/aad.1006

Ce document a été généré automatiquement le 10 décembre 2020.

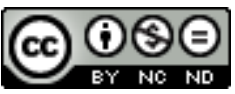

Argumentation \& analyse du discours est mis à disposition selon les termes de la licence Creative Commons Attribution - Pas d'Utilisation Commerciale - Pas de Modification 4.0 International. 


\section{De la paternité biologique à la paternité littéraire : étapes d'une argumentation chez Montaigne}

From Biological to Literary Paternity: The Stages of an Argumentation in Montaigne

Nadine Kuperty-Tsur

\section{NOTE DE L'ÉDITEUR}

Cet article est issu d'une recherche menée dans le cadre de la bourse de l'Israel Science Foundation (GRANT NO. 1223/06).

1 A la différence des autres essais de Montaigne, l'essai 8 du Livre II, intitulé «De l'affection des pères aux enfans ", prend la forme d'une lettre adressée à Louise de La Béraudière, veuve de Louis d'Estissac, qui fut gouverneur de La Rochelle, et mère d'un jeune fils dont elle veille à préserver les intérêts. Les termes dans lesquels Montaigne fait l'éloge du dévouement maternel de la comtesse définissent une relation d'amitié et de respect entre les deux interlocuteurs. Montaigne, ami du défunt mari et magistrat, s'était employé pour les affaires de la comtesse et il se figure ici comme son homme de confiance. Retraçant brièvement l'histoire de la comtesse, Montaigne occupe implicitement une position éthique doublement favorable: il apparaît comme protecteur de la veuve et de l'orphelin, position valorisée par l'ethos chrétien, mais aussi comme substitut de son compagnon d'armes défunt, puisqu'on sait que Louis d'Estissac avait accompagné Montaigne en Italie avant de périr en duel. Montaigne assume donc aussi le devoir à l'ami défunt que dicte la solidarité amicale et noble.

2 Par ailleurs, ce rappel des faits biographiques, bien entendu connus de la comtesse, laisse penser que Montaigne s'adresse également à un tiers, le lecteur, qu'il informe ainsi en quelques mots de la situation de la comtesse et de l'admiration qu'il lui porte. 
Mais le paradoxe de cette lettre tient à ce qu'au bout de deux pages à peine, la comtesse, désignée comme destinataire, disparaît complètement de la situation de communication initiale pour faire place à ses enfants, notamment son fils. Encore n'estil évoqué qu'une fois, brièvement, et son contour est suffisamment flou pour englober le lecteur en général, qui pourrait être assimilé à l'auditoire universel tel qu'il est défini par Perelman et Olbrechts-Tyteca (2008: 41). Montaigne passe, en effet, de l'adresse à la comtesse : «Madame » ou encore " vous », à un « nous » qui désigne, à ce stade, à la fois Montaigne et la comtesse, mais aussi la communauté des hommes. La comtesse se trouve définitivement évacuée du texte qui lui était adressé au moment précis où Montaigne mime un pseudo-dialogue en employant la forme impersonnelle : «Et si on me respond, ce qui fit un jour un seigneur de bon entendements ...» (388). Ce « on » ne marque pas uniquement le passage d'une destinataire précise à un auditoire universel, mais change du même coup le statut même du texte, faisant d'une lettre intime une lettre ouverte et mondaine ainsi que, comme on le verra par la suite, polémique.

Cette lettre figure déjà dans la première édition des Essais, en 1580 (couche A), puis s'augmente des ajouts de Montaigne au fil des éditions successives de 1582 (couche B) et de 1592 (couche $\mathrm{C}$ ). Correspondant au dernier état du texte révisé par Montaigne, la couche $C$ du texte est la plus vindicative et indique que Montaigne confirme, voire renforce, l'opinion qu'il exprimait déjà dans la couche A. Le renforcement du sens au fur et à mesure des éditions montre que Montaigne ne change pas de point de vue, au contraire même, et ce, en dépit du déplacement de la problématique qu'on y constate et qui semble lié au changement des destinataires qu'on vient de signaler.

4 La lettre s'ouvre sur des considérations qui font écho aux arguments de l'avis au lecteur au seuil des Essais, visant à définir le cadre de l'autoportrait très particulier et encore inédit que tente Montaigne et dont il a conscience, puisqu'il le désigne comme «le seul livre au monde de son espece » (II, $8: 386$ ). C'est pour lui une « sotte entreprise » qu'il a du mal à mener à bien et qu'il explique avoir engagée suite au chagrin et à la solitude qu'il éprouvait. Dans l'économie générale des Essais, il est inhabituel qu'un essai adopte la forme de la lettre en mentionnant un destinataire spécifique. Montaigne s'adresse, certes, fréquemment à son lecteur, mais il s'agit d'un lecteur virtuel avec lequel il entretient un rapport de proximité intellectuelle, voire d'amitié dans le style des amitiés humanistes, calqué sur les lettres de Cicéron à Atticus, par exemple, que reprendra Erasme (Fumaroli 1978 : 889). Emaillée de références à Plutarque, à Aristote et à Platon, cette lettre-essai évoque également le style des lettres humanistes dont la vocation était de transmettre un savoir, en tablant sur des valeurs communes, construisant un ethos (Amossy $2006: 69$ ).

Or, l'élaboration de l'ethos dans cette lettre-essai est complexe car, au lieu de renforcer des propositions qu'on pourrait considérer comme " doxiques », Montaigne les attaque tout en tablant sur une communauté de valeurs, partagées avec la comtesse à qui il s'adresse mais sur les deux premières pages uniquement, pour, très rapidement, lui substituer un lecteur virtuel. Reproduisant la dynamique d'une conversation, la lettreessai donne à voir les étapes de la constitution d'une opinion nouvelle, à contrecourant de la doxa en ce qui concerne les relations parents-enfants. C'est bien l'élaboration d'une opinion singulière, dont le cheminement comme les conclusions sont propres à Montaigne et en ce sens le définissent, qui justifie l'insertion de cette lettre, en dépit de sa forme insolite, dans les Essais. Montaigne en a bien conscience puisqu'il prend la peine de se justifier auprès de la comtesse, en expliquant qu'il 
manquerait quelque chose à son autoportrait « si je n'y eusse représenté l'honneur, que j'ai toujours rendu à vos mérites. Et notamment l'amitié que vous aurez montré à vos enfants ». Le dévouement de madame d'Estissac pour ses enfants, le soin qu'elle met à administrer leurs affaires, son refus de contracter une nouvelle alliance, font d'elle un modèle d'amour maternel si rare, que Montaigne veut en laisser un témoignage au fils de cette dame :

Je veux, si ces ecrits viennent un jour à lui tomber en main, lorsque je n'aurai plus ni bouche ni parole qui le puisse dire, qu'il reçoive de moi ce témoignage en toute vérité [...] qu'il n'est gentilhomme en France, qui doive plus à sa mère qu'il fait, et qu'il ne peut donner à l'avenir plus certaine preuve de sa bonté et de sa vertu, qu'en vous reconnaissant pour telle (II, $8: 387$ ).

$6 \quad \mathrm{Au}$ vu de la difficulté et de la déception mutuelle qui marquent les relations parentsenfants que Montaigne analyse dans cet essai, le comportement de la comtesse lui paraît exceptionnel et mérite son éloge, et ce d'autant plus qu'il ne cache pas, par ailleurs et dans la même lettre, sa vision plutôt négative des femmes et de la gestion féminine en général. Mais au moment où il se livre à cette critique, il semble avoir totalement oublié son interlocutrice de départ, il ne la mentionne qu'aux pages 385 et 386 qui débutent cet essai et emploie ensuite la première personne du singulier pour assumer son opinion dans le texte, et la première personne du pluriel quand il évoque une idée ou une valeur à laquelle tous devraient se rallier, comme l'exprime le «nous » dans la phrase suivante: "D'autant que nous avons cher, estre; et estre consiste en mouvement et action » (II, $8: 386$ ).

7 L'argumentation que développe Montaigne s'appuie au début sur l'ethos de l'ami admiratif et respectueux des qualités de la comtesse d'Estissac. Puis cet ethos est rapidement relayé par celui de l'épistolier-essayiste qui se dit à la fois père et magistrat rompu aux affaires de succession. La lettre prend alors un tour philosophique et polémique et pour asseoir sa réflexion, Montaigne s'autorise d'une pratique diversifiée des rapports entre parents et enfants selon les différentes phases de leur existence respective. En mêlant à sa propre expérience une série d'exemples tirés de l'histoire et des sources gréco-latines, Montaigne se livre à l'exposé critique de certaines attitudes parentales afin de promouvoir sa vision de la relation familiale idéale, sinon recommandée. Son analyse se fonde sur des critères d'ordre à la fois affectif et économique et tente d'échapper aux idées reçues en la matière. Sur le plan affectif, il prend dès le départ une position polémique et même anti-doxique dans la mesure où il s'attaque à l'affection généralement éprouvée pour le nourrisson de manière innée, considérant qu'elle devrait plutôt être liée au mérite qu'acquiert l'enfant en grandissant. Par ailleurs, sur le plan économique, Montaigne préconise que la relation parentale passe par le juste partage des ressources matérielles familiales en fonction des besoins respectifs des pères et des enfants. La position anti-doxique annoncée au début de l'essai fera l'objet d'une argumentation visant à démontrer sa pertinence, précisément parce qu'elle ne va pas de soi. Tout se passe comme si Montaigne négociait l'accord de son lecteur en montrant que sa position est en fait, contrairement aux apparences, conforme aux valeurs que tous deux partagent et notamment celles du respect de l'intérêt de l'enfant et de son épanouissement.

8 La particularité de cette lettre-essai tient au fait que les deux pôles de la communication, la posture qu'adopte l'auteur, d'une part, et le profil du destinataire, d'autre part, changent au fil du texte. Le premier pôle désigne la perspective choisie par l'auteur et le parti qu'il défend; or, celui de Montaigne alterne en permanence, 
exposant une fois le point de vue des pères, une autre celui des enfants, pour en définitive créer un " ailleurs » relevant d'un autre ordre qu'on analysera en clôture. En termes d'analyse du discours, la question essentielle qui se pose ici est de déterminer l'effet de l'apparente instabilité des instances de communication sur la capacité persuasive du texte.

9 L'effacement de la destinataire, la première page passée, n'est pas sans effet sur la lettre puisqu'il conduit à deux discours différents: l'éloge personnel et vibrant que Montaigne adresse à cette jeune veuve si dévouée fait place, à la fin de la lettre-essai, à une représentation négative des femmes en général et, plus particulièrement, à l'expression d'une critique acerbe de la gestion féminine des affaires de succession, allant jusqu'à justifier l'application de la loi salique en France. Dès lors, l'évocation aigre des conflits familiaux débouche sur une impasse argumentative qui met en échec l'idéal d'affection, de confiance et de bon sens que Montaigne préconise dans la relation familiale. Montaigne se tire de l'impasse qu'il a lui-même construite en désignant, en clôture de l'essai, une engeance alternative qui court-circuite en quelque sorte la famille dite biologique car, aux œuvres de chair que représentent les enfants, il préférera celles de son esprit avec l'avantage, entre autres, d'en être l'unique géniteur :

il semble qu'il y ait bien une autre production venant de nous, qui ne soit pas de moindre recommandation : car ce que nous engendrons par l'ame, les enfantements de notre esprit, de nostre courage et suffisance, sont produicts par une plus noble partie que la corporelle, et en sont plus nostres; nous sommes peres et meres ensemble en cette generation; ceux cy nous coustent bien plus cher et nous apportent plus d'honeur, s'ils ont quelques chose de bon (Montaigne, II, 8 : 399-400).

Cet essai, souvent évoqué par les historiens, dans le sillage des travaux bien connus de Philippe Ariès sur la notion de l'enfance sous l'Ancien Régime, exprimerait selon eux une attitude emblématique du refus des parents de s'attacher à leurs enfants à cause de la grande mortalité infantile qui touchait au moins un tiers des enfants. Ceci expliquerait certaines phrases de Montaigne, pouvant heurter notre sensibilité contemporaine : "J'ai perdu deux ou trois enfants en nourrice, non sans regrets, mais sans fascherie » (I, 14, 61). On a certes beaucoup glosé sur l'incertitude de Montaigne quant au nombre exact des enfants perdus et l'absence de "fascherie », mais c'est un autre aspect de l'essai qui nous intéresse, notamment la stratégie argumentative qui permet de passer de l'analyse des liens parents-enfants pour, en clôture, proposer une paternité alternative, visant à pallier aux déboires familiaux de toutes sortes que Montaigne passe en revue.

11 On se propose ici de dégager les différentes étapes de la dynamique argumentative du texte pour montrer que cette clôture, surprenante en ce qu'elle déplace complètement les termes du débat proposé, s'inscrit néanmoins déjà dans les prémisses de l'essai. Elle exprime la réticence qu'éprouve Montaigne pour la notion d'amour naturel qu'il trouve bien louche et à laquelle il substituera celles de raison et de jugement parce qu'elles reposent, selon lui, sur des bases à la fois plus dignes de l'homme et surtout plus fiables. Il négocie ainsi sa position quelque peu insolite en l'ancrant dans des valeurs reconnues de tous. 


\section{Contre les lois de la nature}

12 La première partie de cette lettre-essai est consacrée à la critique du bien fondé des « lois de la nature ». Montaigne remarque, certes, que

S'il y a quelque loy vrayment naturelle, c'est-à-dire quelque instinct qui se voye universellement et perpetuellement empreinct aux bestes et en nous (ce qui n'est pas sans controverse), je puis dire, à mon advis, qu'apres le soing que chasque animal a de sa conservation [...], l'affection que l'engendrant porte à son engeance, tient le second lieu en ce rang (II, $8: 386$ ).

13 Mais il ajoute aussitôt, avec lucidité, que cette affection n'est pas réciproque («des enfans aux peres, elle n'est pas si grande »)! Aux lois de la nature, Montaigne oppose le discernement de l'homme qui le distingue des bêtes et lui permet d'exercer son « jugement et liberté volontaire»:

puisqu'il a pleu à Dieu nous doüer de quelque capacité de discours, affin que, comme les bestes, nous ne fussions pas servilement assujectis aux loix communes, ains que nous nous y appliquassions par jugement et liberté volontaire, nous devons bien prêter un peu à la simple authorité de nature, mais non pas nous laisser tyranniquement emporter à elle; la seule raison doit avoir la conduite de nos inclinations (II, $8: 387$ ).

Dès lors que sont posés ces concepts bien humanistes, valorisant la rationalité humaine par opposition à l'instinct animal, la lettre devient le champ d'une démonstration et d'une négociation de valeurs dont on se propose de suivre les étapes et la démarche argumentative. S'attaquant à l'affection innée que les parents sont censés éprouver pour leurs enfants, ce que la doxa considère comme une loi naturelle, la position de Montaigne est catégorique; il ne la partage pas et ne la comprend pas: «je ne puis recevoir cette passion dequoy on embrasse les enfans à peine encore nez, n'ayant ni mouvement en l'ame, ni forme reconnoissable au corps, par où ils puisent se rendre aimables » (II, $8: 387$ ). Et d'ailleurs, il n'a pas pu supporter avoir des nourrissons dans son entourage et le dit sans ambages : «Et ne les ay pas souffert volontiers nourris près de moy » (II, 8 : 387). Montaigne s'inspire vraisemblablement ici du traité de Plutarque, De l'amour et charité naturelle des pères et mères envers leurs enfants, traduit en français par Jacques Amyot en 1549 ; l'ouvrage connaît un grand succès et de nombreuses rééditions au cours $\mathrm{du} 16^{\mathrm{e}}$ siècle, il pose les bases de l'analyse rationnelle du lien parental, démarche qui structure l'argumentation de cet essai.

Si Montaigne s'attaque à l'amour attendri et soi-disant inné qu'éprouvent les parents envers leurs nourrissons, ce n'est pas parce que le mignotage est un peu niais (qui d'entre nous ne s'est jamais penché sur un berceau dans cet état d'esprit?) mais plus précisément, parce qu'à ce stade de leur existence, et selon lui, les nourrissons sont dépourvus des caractéristiques qui permettraient de voir en eux des hommes: « n'ayant ni mouvement en l'ame, ni forme reconnoissable au corps, par où ils puisent se rendre aimables » (II, $8: 387$ ). Philippe Ariès (1973) suggère qu'en ces temps de forte mortalité infantile, les parents, pour se garantir de la douleur de la mort d'un enfant, affichaient une sorte d'indifférence affective qui lui semble bien correspondre à l'attitude de Montaigne. Mais cette thèse est loin de faire l'unanimité parmi les historiens qui ne s'accordent pas non plus sur l'évaluation du pourcentage de décès infantiles - certains parlent de $45 \%$, d'autres de $20 \%$ avant l'âge de 10 ans. Il ne s'agit pas ici de juger Montaigne mais plutôt de comprendre qu'il cherche à surclasser la 
relation parentale en lui conférant une rationalité et une logique spécifiquement humaines, et non instinctives ou encore animales.

\section{lui substituant l'argument d'un amour rationnel parce que fondé sur le mérite des} enfants et donc augmentant en principe au fur et à mesure de leur âge et de leurs actions. De là, il dénonce l'attitude parentale qui consiste à se sentir "plus émus des trépignements, jeux, niaiseries puériles de nos enfants, que nous ne faisons après, de leurs actions toutes formées : comme si nous les avions aimés pour notre passe temps, comme des guenons, non comme des hommes" (II, $8: 387$ ). La démonstration de l'argument est faite dès que Montaigne prouve que l'amour inné, celui qu'il dénonce précisément, est similaire à celui qu'on porte aux petits animaux, pas tant pour eux que pour le plaisir égoïste qu'ils nous procurent. Pour bien faire comprendre le caractère indigne de cette affection, Montaigne la compare à celle que suscitent des " guenons ». Cette image fonctionne comme un argument qui tire sa force persuasive de la réduction explicite de l'homme à l'animal, image efficace dès lors qu'elle choque. Car qui accepterait de s'entendre dire qu'il aime ses enfants comme on aime des petits singes? L'ethos humain s'en indigne et c'est bien sur ce mouvement d'indignation que table Montaigne pour expliciter son point de vue et convaincre de sa pertinence. Cette comparaison dégradante fait d'emblée apparaître que la dimension qui doit être revendiquée pour nos enfants est autre, liée à l'intellect et au spirituel, valeurs spécifiquement humaines.

Passant du stade du nourrisson à celui de l'enfant qu'on éduque, Montaigne constate qu'« un tel fournit bien liberalement de jouets à leur enfance, qui se trouve resseré à la moindre despense qu'il leur faut estant en aage » (II, $8: 387$ ). Autrement dit, il établit une hiérarchie claire entre les investissements nécessaires aux différents stades de la croissance. Son discours, lucide et aussi complet que possible, envisage les multiples aspects de la relation parentale en s'affranchissant des lieux communs de l'époque. Ses réflexions visent à acquérir la force de l'évidence et peut-être est-ce aussi la raison du passage d'un destinataire concret et privé à un lecteur virtuel et universel. Lorsqu'il évoque, à ce titre, une dimension existentielle, inhérente à l'inévitable compétition entre les générations : « il semble que la jalousie que nous avons de les voir paroistre et jouyr du monde, quand nous sommes à mesme de le quitter, nous rende plus espargnans et rétrains envers eux : il nous fache qu'ils nous marchent sur les talons comme pour nous solliciter de sortir» (II, $8: 387$ ), ce «nous » ne désigne plus la comtesse et Montaigne mais bien la communauté des parents face à celle des enfants, « eux ».

La jeunesse, ce bien inégalement réparti, dont les uns jouissent au moment où les autres en sont privés, conduit l'argumentation, par association d'idées, vers un autre aspect de la relation parentale, auquel Montaigne accorde une extrême importance : celui de la répartition des biens matériels. C'est précisément au moment où il développe ce sujet qu'il change de point de vue et passe de celui des parents à celui des enfants. 


\section{Changement de point de vue: Montaigne, avocat des enfants contre leurs pères}

19 Jusqu'ici, Montaigne occupait la position de l'observateur neutre, ou se plaçait plutôt du côté des pères, mais lorsqu'il aborde la dimension matérielle de la relation parentale, il se pose en défenseur des droits des enfants et se fait le porte-parole de leurs besoins. Le changement de point de vue s'opère à partir d'une phrase-pivot qui souligne la dépendance des enfants : « l'ordre des choses porte qu'ils ne peuvent, à dire verité, estre ny vivre qu'aux dépens de nostre estre et de nostre vie [...]» (II, $8: 387)$. Il s'agit bien ici d'un vif débat autour de valeurs qui engagent la responsabilité morale des parents. Montaigne ne mâche pas ses mots et dénonce comme

cruauté et injustice de ne les recevoir au partage et société de nos biens, et compaignons en l'intelligence de nos affaires domestiques, quand ils en sont capables, et de ne retrancher et resserer nos commoditez pour pourvoir aux leurs puisque nous les avons engendrez à cet effect (II, $8: 388$ ).

L'argument du partage des biens s'appuie sur plusieurs exemples concrets et d'autant plus convaincants qu'ils ont le statut de témoignages de première main issus de l'expérience vécue de Montaigne. Il montre les dégâts que cause l'avarice des pères, poussant les enfants au vol, habitude dont ils sont ensuite incapables de se défaire :

On les jette au desespoir de chercher par quelque voie si injuste qu'elle soit, à pourvoir à leur besoing : comme j'ai veu de mon temps plusieurs jeunes hommes de bonne maison, si adonnez au larcin, que nulle correction les en pouvoit détourner (II, $8: 388$ ).

21 De même, défendant l'intérêt des enfants, il ne cache pas son mépris pour les pères qui abusent de la dépendance matérielle de leurs enfants pour se faire respecter : «Un père est bien miserable, qui ne tient l'affection de ses enfans que par le besoin qu'ils ont de son secours, si cela se doit nommer affection » (II, $8: 389$ ). Ces exemples d'usage abusif de l'autorité paternelle préparent le terrain à l'exposé des valeurs de Montaigne en matière d'éducation : il condamne la violence et les châtiments, contre-productifs si on veut éduquer à l'honneur et à la liberté, argument qu'il illustre à partir de l'éducation qu'il a lui-même reçue et qu'il a dispensé ensuite à sa fille et qu'il estime «juste et naturelle ». On notera qu'ici le naturel qu'il stigmatisait au début de son essai, prend une connotation positive et a presque valeur d'évidence: l'affection étant perçue comme un lien plus authentique que celui du besoin matériel. Par ailleurs, cet exemple sert de nouveau pivot de l'argumentation et permet à Montaigne de passer du point de vue de l'enfant, lorsqu'il décrit le type d'éducation qu'il a reçue, au point de vue du père, lorsqu'il assume vis-à-vis de sa fille le rôle d'éducateur.

\subsection{Montaigne et le point de vue du père}

\subsection{La dimension matérielle de la relation parentale comme point de départ de l'enquête et de l'argumentation}

Comment faire pour être aimé de ses enfants et que, bien qu'avides d'hériter, ils ne souhaitent cependant pas notre mort? Pour le coup, cette franchise peut étonner mais il faut reconnaître que ce ton direct détermine à la fois la crédibilité tout comme l'intérêt du débat. Car, en mettant cette question au centre de sa démarche, Montaigne met bas les masques et montre l'authenticité de sa réflexion qui s'affranchit des 
stéréotypes et des convenances. Il ne s'agit pas d'un exercice de rhétorique sur des cas d'école mais d'un vrai débat de société en prise sur un malaise réel, éprouvé par les enfants comme par les parents et qui se nourrit clairement de l'expérience juridique de Montaigne (Basalmo 2004). Le conflit des générations est décrit à partir des domaines affectifs, matériels ainsi que celui de la responsabilité morale. En gros, Montaigne, adoptant à présent le point de vue des pères, essaie de définir des critères et des limites. Pour éviter les conflits d'intérêts entre les besoins des enfants pour entrer dans le monde et ceux des parents, encore jeunes et en train de se faire un nom: "un gentilhomme qui a trente cinq ans, il n'est pas temps qu'il face place à son fils qui en a vingt : il est luy-mesme au train de paroistre et aux voyages des guerres et en la court de son Prince » (II, $8: 390$ ), il faut donc qu'entre le père et le fils, il y ait une différence d'âge d'au moins 35 ans. Ce sont d'ailleurs les recommandations d'Aristote et Platon que Montaigne évoque pour accréditer son opinion.

Rien d'étonnant comme on le comprendra mieux à la fin de cet essai, que ce soit Thalès, le vrai sage aux yeux de Montaigne qui «Jeune, respondit à sa mere le pressant de se marier, qu'il n'estoit pas temps; et devenu sur l'aage, qu'il n'estoit plus temps » (II, 8 : 390)! Ne recommandant ni le mariage ni la fondation d'une famille, Montaigne la considère au contraire comme un handicap à l'énergie masculine, l'empêchant de s'investir ailleurs. Il évoque l'abstinence bénéfique des champions grecs, tout comme l'opinion des Gaulois qui considèrent que l'acte sexuel amollit l'homme et le contraint à consacrer ses forces vitales à la nourriture de sa progéniture.

Sensible aux besoins parfois contradictoires des pères et des fils, Montaigne poursuivant toujours l'argument économique, préconise aux pères de se dépouiller dans la limite de leurs intérêts : « non pas jusques à la chemise, mais jusques à une robe de nuit, bien chaude » (II, $8: 391$ ). La sagesse étant de savoir ce qui convient à son âge, autrement dit savoir quand se retirer pour laisser la place aux enfants en âge de paraître, en s'arrangeant toutefois pour que le don ne soit pas irréversible au cas où les enfants dérogeraient - la satisfaction du père étant de constater qu'il peut passer la main tout en servant de guide à ses enfants afin de voir l'ordre de la maison se poursuivre. L'idéal serait que les parents âgés vivent à proximité de leurs enfants, profitant de leurs joies mais sans les gêner par les incommodités de leur âge. Ce point n'est pas énoncé comme une vérité générale, somme toute assez banale, mais tire sa force de ce qu'il est revendiqué sur le mode personnel, dévoilant ainsi les vœux (inquiets) de Montaigne et conférant également à l'argument le poids d'un sentiment authentique: «je voudroy les esclairer de pres, et jouyr, selon la condition de mon aage, de leur allegresse et de leurs fêtes. Si je ne vivoy parmi eux [...], je voudray au moins vivre pres d'eux » (II, $8: 392$ ).

Montaigne emploie différentes stratégies, passant de l'inscription subjective à une position d'analyste objectif lorsqu'il tente de concilier son point de vue de père avec les revendications des enfants et c'est à nouveau leur point de vue qu'il défend lorsqu'il multiplie les témoignages de déchéances pathétiques de vieux pères s'entêtant à gouverner leur monde en se couvrant de ridicule, sans même s'apercevoir qu'on les trompe et vole de toute part. La force de l'argumentation tient ici à la justesse de l'observation, issue d'une expérience qui est à la fois présentée comme commune à tous mais qui se nourrit également d'une pratique juridique, liée à l'activité de Montaigne comme magistrat au parlement de Bordeaux. La force persuasive de l'argument tient encore à une critique qui en appelle au bon sens, et à la volonté implicite de trouver 
une solution objective, c'est-à-dire équitable pour les enfants tout en préservant la dignité des pères, en vue d'un fonctionnement social harmonieux.

L'art de Montaigne tient dans l'agencement habile de ses arguments : ses descriptions de situations diverses débouchent sur des analyses fines destinées à susciter l'adhésion du lecteur censé se rallier à la logique, au bon sens mais aussi à la complexité des analyses. D'un trait sûr, il va à l'essentiel et les solutions qu'il préconise semblent s'imposer d'elles-mêmes, sans qu'il soit nécessaire d'user d'autorité. La force persuasive du discours vient aussi de ce qu'étant une fois du côté des parents, et une autre du côté des enfants, le locuteur se crée ainsi un lieu d'énonciation objectif qui lui permet de s'ériger en juge impartial, appelant tout lecteur un tant soit peu rationnel de se rallier à son argument.

\subsubsection{La dimension affective}

Dénonçant tout ce qui relève de l'abus d'autorité parentale, Montaigne s'insurge contre les pratiques et les modes en vigueur dans la noblesse de son époque, tel le vouvoiement que les nobles exigeaient de leurs enfants qui devaient les appeler monsieur mon père, madame ma mère, pour marquer leur respect, leur infériorité, pour exprimer leur situation de dépendance. Attitude que Montaigne dénonce comme abusive bien sûr, mais surtout comme inhibant toute possibilité de relation affective authentique. Lucide, il avertit que le temps joue en faveur des enfants et en défaveur des pères en les rendant ridicules, ainsi son choix est fait : "Quand je pourroy me faire craindre, j'aimeroy encore mieux me faire aymer " (II, $8: 393$ ). Idée qu'il répète à plusieurs reprises et à la première personne pour montrer à quel point il l'incarne : "J'essayeroy, par une douce conversation de nourrir en mes enfans une vive amitié et bienveillance non feinte en mon endroit [...]» (II, $8: 392$ ). La proximité et l'affection sont incompatibles avec la morgue "austère et dédaigneuse " que certains pères, " vrais espouvantails de cheneviere » (!) affichent pour se faire obéir et que Montaigne dénonce comme une «farce très inutile qui fait haïr les pères aux enfants ». Il a recours à des images fortes pour exprimer le ridicule de ces attitudes paternelles tyranniques et le peu d'effet qu'elles ont sur les enfants qui s'en moquent d'ailleurs car «Ils ont la jeunesse et les forces en main, et par consequent le vent et la faveur du monde; et reçoivent avecques mocquerie ces mines fieres et tyranniques d'un homme qui n'a plus de sang ny au cœur, ny aux veines, vrais espouvantails de cheneviere » (II, $8: 393$ ).

Mêlant le particulier au général, Montaigne s'appuie sur des situations issues de son expérience mais qui fonctionnent comme des études de cas typiques, mettant en scène des pères et des enfants, le plus souvent sans nom et sans visage. Mais cet anonymat ne met pas en cause la crédibilité des exemples puisqu'il vise à préserver l'intimité des personnages évoqués. L'anonymat fonctionne, au contraire comme une preuve indirecte de leur vérité historique. Montaigne peut également adopter la technique opposée qui consiste, par exemple, à évoquer un personnage connu de tous et dont l'expérience singulière frappera d'autant plus le lecteur qu'il en identifie le protagoniste. C'est le cas de l'exemple étonnant du maréchal Blaise de Monluc et de son fils tué au front. Ces deux personnages historiques confèrent à l'exemple jusqu'ici général et anonyme, une corporalité, au sens que Dominique Maingueneau donne à ce terme. Contemporain des guerres de religion (1562-1598), dont la dernière décennie recoupe les années successives de parution des Essais (1580-1588-1592), le lecteur des Essais voit en Monluc, l'homme de guerre, intransigeant et cruel, responsable de la 
répression sanglante des protestants dans le Sud-Ouest de la France (Constant 2002). Cruauté telle qu'elle conduira Charles IX à désavouer ce maréchal et à le renvoyer sur ses terres, en dépit de l'horrible blessure reçue au siège de Rabastens, qui l'a défiguré et l'oblige à porter un masque de fer. L'évocation d'un entretien privé entre Montaigne et Monluc sur son comportement paternel est surprenante et, de là, persuasive (Amossy, 2006 : 206), on y découvre chez ce bourreau notoire un homme capable de sentiment. L'affectivité et le regret pathétique qui émanent de son aveu dévoilent une dimension insolite du personnage et confèrent à l'anecdote une présence à la fois authentique et poignante. Père endeuillé, Monluc ne se pardonnera jamais d'avoir montré à son fils un visage injustement sévère :

Et ce pauvre garçon, disoit-il, n'a rien veu de moy qu'une contenance refroignée et pleine de mespris, et a emporté cette creance que je n'ay sceu ny l'aymer, ny l'estimer selon son merite. A qui gardoy-je à découvrir cette singuliere affection que je luy portoy dans mon ame ? estoit ce pas luy qui en devoit avoir tout le plaisir et toute l'obligation? Je me suis contraint et geiné pour maintenir ce vain masque ; et y ai perdu le plaisir de sa conversation, et sa volonté quant et quant, qu'il ne me peut avoir portée autre que bien froide, n'ayant jamais reçeu de moy qu rudesse, ny senti qu'une façon tyrannique (II, $8: 395$ ).

L'aveu que livre Monluc, dans ce discours rapporté à la première personne, est d'autant plus dramatique que son fils est mort au combat avant même qu'un dialogue véritable ait pu s'établir entre eux. La mort du fils rend la situation irréversible: il ne saura jamais que son père l'aimait. Cet épanchement insolite dans la bouche du rude soldat (voire du bourreau de protestants) qu'était Monluc est émouvant à la fois par sa sobriété, sa sincérité et sa dimension pathétique. Monluc est ici en quelque sorte "ventriloqué» par Montaigne qui l'utilise comme pièce maîtresse de son argumentation, objectivée de fait par l'exemple d'un tiers.

\section{Les cautions argumentatives : l'insertion du « je », le statut des témoignages}

Montaigne s'implique dans son texte de plusieurs façons: il fait part de sa propre expérience, on l'a vu, l'éducation qu'il a reçue lui sert de modèle, le récit d'exemples qu'il a pu observer autour de lui, comme témoin direct est souligné par une incise personnelle forte :

J'ai veu encore [...] Et ay connu tel Seigneur [...] (396) ; J'en voy envers qui [...] J'en connois un qui [...] (388); Je me mariay à trente trois ans $(390)$; j'ay veu de mon temps et connu familierement des personnages de grande authorité (391); J'en ay veu quelqu'un [...] (393); Quand j'oy reciter l'estat de quelqu'un, je ne m'amuse pas à luy ; je tourne incontinent les yeux à moy, voir comment j'en suis $(395) ;[. ..] \mathrm{Je}$ m'ouvre aux miens (396).

31 La fréquente insertion de l'auteur à la première personne ajoute une caution personnelle aux exemples de sa démonstration. Montaigne multiplie les preuves et les emprunte à toutes sortes de registres : expérimental, on l'a vu, issu de l'observation personnelle et directe de son entourage mais aussi livresque par les nombreuses références aux anciens (notamment p. 400 et 401) et historiques enfin, évoquant pêlemêle, les Gaulois, Charles V et Monluc. Montaigne traite ces exemples à l'identique, comme une série de témoignages venant étayer son opinion, sans égard particulier pour leur différence de statuts ou de contextes. Il est cependant conscient du 
conditionnement culturel et historique de la relation parentale comme le montre l'exemple qu'il donne des Gaulois, rapporté par Jules César qui observait que ce n'est qu'à l'âge où les enfants portaient des armes qu'ils se rapprochaient de leurs pères (II, $8: 396)$.

\section{Contre les femmes}

Alors que cette lettre s'ouvrait sur une adresse explicite à la comtesse d'Estissac et sur l'éloge de son comportement à l'égard de son fils, Montaigne semble oublier sa destinataire, dès la seconde page. Il s'adresse, on l'a vu, au lecteur (masculin) en général, assimilable à un auditoire universel. Ce changement de destinataire rend possible la vision négative et stéréotypée des femmes, "déraisonnables par principe " et incapables de gérer les biens familiaux, qu'on trouve dans la dernière partie de l'essai. Montaigne abandonnant le point de vue objectif qui le caractérisait, prend à présent clairement le parti masculin en se jetant dans la guerre des sexes : «Il est tousjours proclive aux femmes de disconvenir à leurs maris : Elles saisissent à deux mains toutes couvertures de leur contraster » (II, $8: 394)$. Il considère encore que le fait qu'une femme gère le bien laissé par le père témoigne d'un défaut d'éducation : à savoir que les enfants ne sont pas aptes à gérer le bien familial. Les chefs d'accusation contre les femmes sont nombreux : elles dérobent l'argent de leur mari pour faire de grasses aumônes à leur confesseur, usurpent l'autorité paternelle et complotent contre lui, disposent des héritages qu'elles dilapident, etc.

Parmi ces accusations en bloc, Montaigne distingue cependant le cas des mères, en dépit de «l'ordinaire foiblesse du sexe » et admet qu'il serait (tout de même) « contre nature de faire dépendre les meres de la discrétion de leurs enfans» (II, $8: 397$ ). Revenant sur la nécessité du partage des biens entre parents et enfants, il stigmatise le père qui monopolise ses biens puis ensuite la mère qui, lorsqu'elle en hérite, ne les partage pas. Lorsqu'enfin le fils reçoit cette fortune, il est souvent trop tard pour qu'il puisse la mettre à profit et construire sa carrière. Évoquant les existences gâchées par l'égoïsme des parents, Montaigne soulève ici un problème capital pour la société de son temps, à savoir le manque d'avenir des jeunes nobles, entièrement dépendants de la bonne volonté de leurs parents pour faire carrière, acheter un équipement, payer des troupes, ou simplement acquérir une éducation.

En dernier recours, c'est à la sagesse des lois et à la tradition que s'en remet Montaigne pour ordonner la gestion des biens et sortir de l'impasse, à la fois familiale et argumentative sur laquelle son analyse achoppe : «En general la plus saine distribution de nos biens en mourant, me semble estre, les laisser distribuer à l'usage du païs. Les loix y ont mieux pensé que nous » (II, $8: 397)$.

Procédant sur le mode associatif, l'idée de la défaillance du jugement et du palliatif que représentent les lois l'amène à évoquer le jugement des femmes, gouvernées par leur sens, comme des animaux, ce qui justifie, selon lui, la loi salique, appliquée uniquement en France et « qui prive les femmes de la succession de la couronne ». Montaigne passe donc du domaine familial au domaine politique pour prononcer, dans des termes sans appel, l'incompétence des femmes. Mais il les met également en scène de façon tout aussi problématique, dans un domaine ordinairement valorisé et qui est exclusivement le leur, celui de l'allaitement. A ce stade de sa démonstration, Montaigne revient sur la critique des lois de la nature qui ouvrait cet essai, en dénonçant le marché qu'acceptent 
les nourrices qui, pour de l'argent, allaitent les enfants des autres, aux dépens des leurs, parvenant même à transférer leur affection sur l'enfant étranger. L'argument vise donc à les peindre en mères doublement monstrueuses, appâtées par le gain d'une part et coupables d'un amour " contre-nature " pour l'enfant étranger qu'elles allaitent, amour contre-nature du fait qu'il met le leur en danger de mort. Sans quitter ce terrain, Montaigne poursuit avec une étrange anecdote racontant que, dans sa région, on a parfois recours aux chèvres pour remplacer les nourrices défaillantes et, tout comme les femmes, les chèvres s'attachent aux enfants qu'elles nourrissent. Loin d'être anodine, cette anecdote opère une confusion intentionnelle entre allaitement et attachement, afin de mettre sur le même plan l'attachement des mères et celui des chèvres sous le seul prétexte que les unes comme les autres allaitent. De là, il n'y a qu'un pas que Montaigne franchit allègrement pour reprendre l'argument développé en ouverture de cet essai, et conclure à présent que l'attachement dit «naturel » pour l'enfant n'est en fait qu'un instinct bestial. L'attachement (ce que la doxa valorise et appelle plutôt l'amour maternel) sert paradoxalement de base à la comparaison dégradante entre les femmes et les chèvres, replaçant l'affection maternelle, par le biais de l'allaitement, dans le contexte animal et biologique et ce, en dernier lieu pour stigmatiser les lois de la nature et les femmes.

Ainsi, la cohérence tout comme la cohésion de la démonstration dans cet essai sont assurées par un argument en boucle. Montaigne débutait par une attaque contre les lois dites naturelles, en montrant qu'elles reniaient l'humanité de l'enfant, il y revient à la fin de l'essai, sous un autre biais, celui de l'allaitement, pour dénoncer la dimension affective, naturelle et valorisante qu'on lui confère d'ordinaire. Il attaque le lieu commun liant l'affection à l'allaitement en montrant que ce lien dit « naturel » n'en est pas moins fragile: il ne résiste ni à l'appât du gain, ni à l'habitude puisque dans les exemples évoqués par Montaigne, l'amour naturel lié à l'allaitement fait place à une sorte de réflexe automatique et se trouve donc dépourvu de toute valeur humaine. A l'instar des chèvres, les femmes adoptent sans discernement l'enfant qu'elles allaitent. Montaigne, certes, ne le dit pas, mais poussé à bout, l'argument qui débutait en discréditant la capacité de gestion des femmes se poursuit en détruisant le mythe de l'attachement naturel lié à l'allaitement en mettant les femmes et les chèvres sur un même plan. Parce que l'allaitement relève du domaine animal, le fait que les femmes allaitent ne les qualifient donc pas pour gérer un patrimoine au sens où cela ne garantit en rien leur capacité à prendre soin des intérêts (pris au sens large) de l'enfant.

\section{La paternité alternative}

Cette lettre de Montaigne, destinée à l'origine et sur deux pages seulement à la comtesse d'Estissac, donne une vision plutôt pessimiste de la relation familiale en général. Rien d'étonnant donc à ce que Montaigne, au terme de cette revue grinçante de la relation parentale et devant son impasse presque structurelle, propose une alternative ou encore une nouvelle définition de la paternité (Kritzman 2009 : 121-122) qui accorde plus de valeur aux « enfantements de son esprit » qu'à ses propres enfants (Woods 2003). Non sans humour, Montaigne finit par considérer qu'engendrées par une partie plus noble, ces œuvres seront des enfants immortels (et peu dérangeants), susceptible de lui attirer une gloire durable, voire éternelle et en tout cas exclusive. En marge de l'ironie, émane aussi sous ce semblant de question qu'il s'adresse, une bonne 
dose de scepticisme quant aux rapports hommes-femmes: «Et je ne sçay si je n'aimerois pas mieux beaucoup en avoir produict ung [enfant], parfaictement bien formé, de l'acointance des muses, que de l'acointance de ma femme » (II, $8: 401)$. Les divers accomplissements des hommes représentent tous des «filiations" plus ressemblantes à leur auteur que les enfants à leur père et, en ce sens, plus conformes au projet d'autoportrait de Montaigne: "Car la valeur de nos enfans est beaucoup plus leur que nostre; la part que nous y avons est bien legiere; mais de ceux cy toute la beauté, toute la grace et pris est nostre. Par ainsi, ils nous representent et nous rapportent bien plus vivement que les autres » (II, 8: 400). La force de cette autre paternité est illustrée par une quantité d'exemples d'auteurs, tels Labienus et Greuntius Cordus, qui choisirent de mourir ne pouvant surmonter de voir leurs écrits («si chere géniture ») brûlés :

Or Labienus ne peut souffrir cette perte, ny de survivre à cette sienne si chere geniture (II, $8: 400)$.

Pareil accident advint à Greuntius Cordus, accusé d'avoir en ses livres loué Brutus et Cassius. Ce senat vilain, servile et corrompu, et digne d'un pire maistre que Tibere, condamna ses escripts au feu ; il fut content de faire compagnie à leur mort, et se tua par abstinence de manger (II, $8: 401$ ).

De même, Lucanus, sur son lit de mort, récite des vers de son livre à l'instar d'un père disant adieu à ses enfants: "Cela, qu'estoit ce qu'un tendre et paternel congé qu'il prenoit de ses enfans? ? (II, $8: 401)$

Il ne suffit pas à Montaigne d'avoir démontré l'existence d'une paternité alternative, (il se prendra d'ailleurs à la fin de sa vie d'une authentique affection pour Marie le Jars de Gournay, sa fille spirituelle) mais il consacre la clôture de son essai à négocier sa primauté sur la paternité biologique en posant une série de pseudo-questions mettant en jeu l'ethos humaniste renaissant : existe-t-il un poète qui aurait préféré être père "du plus beau garçon de Rome ", que de l'Eneide ? Epaminondas aurait-il jamais accepté d'échanger les deux victoires remportées sur les Lacédémoniens pour «les plus gorgiases de toute la Grèce "? De même César puis Alexandre et enfin le sculpteur Phidias qui sert d'habile transition pour évoquer Pygmalion et avec lui la passion qu'un créateur peut éprouver pour son œuvre, à tel point « qu'il fallut qu'en faveur de sa rage les dieu la luy vivifiassent $»$.

\section{Conclusion}

C'est dans ce contexte pessimiste général qu'on comprend mieux le caractère remarquable du comportement de Madame de L'Estissac et la volonté de Montaigne, impressionné au point de vouloir en témoigner dans cette lettre-essai, non pas tant pour la comtesse, puisque nul n'est mieux informée qu'elle de sa propre vie et de ses sacrifices mais bien pour ses enfants, pour la reconnaissance de cette mère d'exception. L'effacement de la comtesse de la liste des destinataires s'impose donc pour extirper la lettre du circuit de l'amicale intimité et en faire une véritable lettre-ouverte, célébrant à la face d'un tiers, le fils et la postérité en général, son comportement si exceptionnel au vu de la complexité de la relation parentale.

41 Mais dans cette curieuse «lettre-essai» qui se libère du cadre épistolaire initial, Montaigne poursuit avant tout un but argumentatif clair et cohérent : celui de remettre en question la notion de lois naturelles et plus précisément d'affection naturelle, pour à 
partir de là sonder la complexité de la relation parentale et d'en dégager, en s'appuyant sur des sources dont la diversité participe du pouvoir de conviction, une vision nouvelle. A l'issue de ce parcours, Montaigne aura négocié les valeurs de l'esprit contre celles de la nature, les œuvres de papier contre celles de chair, marché reposant tout entier sur les valeurs de l'humanisme renaissant et de son admiration sans borne pour l'Antiquité et les Belles Lettres comme preuves de la capacité de l'homme à atteindre au sublime. S'il parvient à convaincre de positions " anti-doxiques ", c'est en faisant appel à l'ethos humaniste duquel se réclame le lecteur qu'il construit en le substituant à sa première destinataire. Cet ethos se définit par opposition à l'animal, femelle de préférence (cf. les guenons et les chèvres). Ainsi aux lois de la nature, assimilées à la bestialité et à l'instinct, Montaigne substitue la réflexion, les constructions ou créations de l'esprit, spécifiques à l'homme, définissant à la fois sa particularité et sa dignité.

C'est ainsi que le texte, qui débute comme une lettre intime, avec le changement de destinataire change de statut et devient une lettre mondaine à visée critique puisque son auteur y anime un débat qui, minant les positions de la doxa, œuvre sur la base des valeurs humanistes pour suggérer une nouvelle définition de la paternité issue de l'esprit, exclusive mais aussi, de son point de vue, supérieure.

\section{BIBLIOGRAPHIE}

Amossy, Ruth. 2006. L'argumentation dans le discours (Paris : Armand-Colin)

Ariès, Philippe. 1973. L'enfant et la vie familiale sous l'Ancien Régime (Paris : Seuil)

Basalmo, Jean. 2004. « La critique des dispositions testamentaires : un scepticisme peu philosophique ", Demonet, Marie Luce \& Alain Legros (éds.). L'écriture du scepticisme (Genève : Droz), 275-287

Constant, Jean-Marie. 2002. Les Français pendant les guerres de religion (Paris : Hachette)

Fumaroli, Marc. 1978. « Genèse de l'épistolographie classique », Revue d'Histoire Littéraire de France $6,886-900$

Kritzman, Lawrence. 2009. The Fabulous Imagination. On Montaigne's Essays (New York: Columbia University Press)

Montaigne, Michel de. 2004. Les Essais t. II, éd. par Pierre Villey (Paris : Quadrige /PUF)

Perelman, Chaïm \& Lucie Olbrechts-Tyteca. 2008. Traité de l'argumentation (Bruxelles : éditions de l’Université de Bruxelles).

Plutarque. 1549. " De l'amour et charité naturelle des pères et mères envers leurs enfants ", Les œuvres morales et philosophiques, traduit par Jacques Amyot (Paris)

Viala, Alain. 1981. « La genèse des formes épistolaires en français et leurs sources latines et européennes. Essai de chronologie distinctive (XVIe -XVIIe siècle) ", Revue de littérature comparée $218,168-183$ 
Woods, Robert. 2003. " "Did Montaigne Love His Children?" Demography and the Hypothesis of Parental Indifference », Journal of Interdisciplinary History 3:3, 421-442

\section{RÉSUMÉS}

Dans « De l'affection des pères aux enfants ", Montaigne, à la fois père et magistrat rompu aux affaires de succession, entame une réflexion critique sur les rapports entre parents et enfants. L'analyse qu'on propose ici part de la lettre à la comtesse qui constitue le premier noyau de l'essai, pour retracer l'argumentation du locuteur-scripteur qui va de la valeur de l'amour maternel à celle de l'enfantement de l'œuvre (de l'écrivain) en passant par l'amour paternel. On montre comment la négociation des valeurs est déplacée dès lors que le discours monogéré de l'essai se substitue à la lettre: l'adresse à l'autre est effacée, et l'essai propose une nouvelle définition de la paternité.

In his essay "On the paternal affection for children" Montaigne (himself a father and a magistrate greatly experienced in succession cases) proposes a critical revision of the topos of the relations between parents and children. The analysis we submit here starts with the letter to the Countess, first nucleus of the essay. We then try to show how the argumentation proceeds, from the value of maternal love to that producing forth a literary/philosophical work, with paternal love as the intermediary link. The negotiation of values comes to an end when the monological discourse of the essay is substituted for the dialogical discourse of the correspondence: the address is obliterated, and the essay offers a new definition of fatherhood.

\section{INDEX}

Mots-clés : Les Essais (Montaigne), loi naturelle, misogynie, paternité biologique, paternité spirituelle

Keywords : biological paternity, Essais (Montaigne), misogyny, natural law, spiritual paternity

\section{AUTEUR}

\section{NADINE KUPERTY-TSUR}

Université de Tel Aviv 